\title{
Electron Microscopy Studies Superconducting BaMX, Family Materials
}

Cigdem Ozsoy-Keskinbora ${ }^{1}$, Aravind Devarakonda ${ }^{2}$, Austin Akey $^{3}$, Stephan Kraemer ${ }^{3}$, Joseph Checkelsky $^{2}$, David C. Bell ${ }^{1}$

${ }^{1}$ Harvard John A. Paulson School of Engineering and Applied Sciences, Harvard University, Cambridge, MA, USA

2. MIT, Department of Physics, , Cambridge, MA, USA

${ }^{3}$ Center for Nanoscale Systems, Harvard University Cambridge MA, USA

1911 was an important milestone due to the realization of abrupt resistivity drop on solid mercury at cryogenic temperatures. This discovery known as superconductivity opened up an important era in solid state research. Still, today it is one of the most investigated fields for its applicability in MRI/NMR machines, mass spectrometers, particle accelerators, SQUIDs, RF and microwave filters, quantum computing, etc. Usage of the superconductivity ether by itself or combination with other exotic topological phenomena in quantum computing creates the needs for investigation not only already known materials system but also new materials that the superconductivity can be tuned, modified or controlled.

The exploration and synthesis of constitute only one aspect of the challenges in the development of new materials, another challenge is their characterization. Since the phenomena appear at very restricted and dedicated conditions, the characterization method must have very high sensitivity, resolution, localization, and precision. Since, the electromagnetic properties of the superconductors, determined by the crystal structure and composition, to be able to understand, analyze and control these properties, both crystal structure and composition need to be known precisely. Transmission electron microscopy is a powerful technique to investigate structural, compositional or electromagnetic properties of materials. Especially, the recent implementation of aberration correction [1] in the transmission electron microscopy made chemical and structural characterization with very high spatial resolution and sensitivity possible [2]. This, in turn, allows for spatial resolution in the range of picometers for characterization of superconductor and topological materials, where small compositional variations have large effects on the material properties

Here, in this study, we summarize detailed electron microscopy studies on $\mathrm{BaNbS}_{3}$ which was synthesized as an inspiration by a 2016 theoretical study proposes that $\mathrm{BaMX}_{3}$ crystal family can support topological semimetal state [3]. The findings of STEM, atomic resolution (S)TEM, EELS (figure 1), EDX experiments for compositional, structural and excitonic properties of the material will be reported. 
References:

1) Rose, H., J. Electron Microsc. 2009, 58, 77-85

2) Krivanek, O. L. et al., Nature 2010, 464, 571-574

3) Liang, Q. et al., Phys. Rev. B, 93, 085427

4) This work was supported by the STC, Center for Integrated Quantum Materials, NSF Grant No. DMR-1231319

5) This work was performed at the Center for Nanoscale Systems (CNS), a member of the National Nanotechnology Coordinated Infrastructure Network (NNCI), which is supported by the National Science Foundation under NSF award no. 1541959.
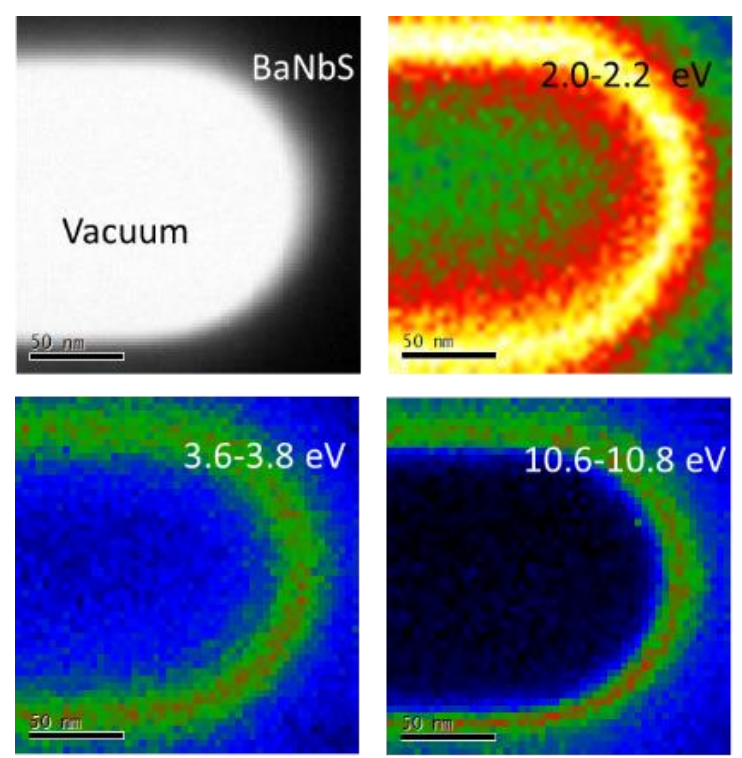
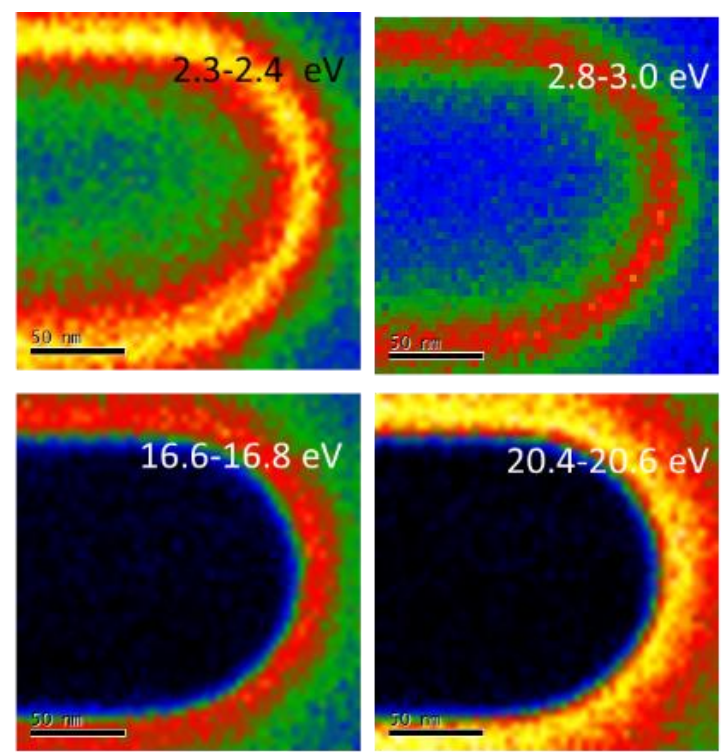

Figure 1. EFTEM images of focused ion beam structured BaNbS in electron energy loss range from 2.0 to $20.6 \mathrm{eV}$ with $0.2 \mathrm{eV}$ energy steps. 